\title{
Sex and the Military: A model for provider education
}

\author{
Sherrie L. Wilcox, Doni P Whitsett and Kimberly Finney* \\ University of Southern California, USA
}

\begin{abstract}
Military populations have increased exposure to physical and psychological trauma, which increases their vulnerability to sexual dysfunction. Despite high rates of sexual functioning problems in military populations, including erectile dysfunction and anorgasmia, few behavioral health providers are adequately prepared to address these problems, partly due to a lack of training options. The Sex \& the Military Toolkit is the first freely available training on sexual dysfunction in military populations that provides preliminary insight on this key issue and is translatable to civilian populations. This manuscript presents an overview of the toolkit with emphasis on the video vignettes (confabulated) and provides clinical considerations, therapy suggestions, and sex-specific intervention strategies.
\end{abstract}

\section{Sex and the Military: A model for provider education}

Sexual functioning is a key part of sexual health [1,2] and an integral part of life [3]. Sexual dysfunction is associated with disruption in multiple areas of functioning, including psychological, social, and physical [4]. Military populations are vulnerable to sexual dysfunction due to an increased risk of mental and physical health problems, including posttraumatic stress disorder (PTSD), depression, and lower-body physical trauma [5].

During the past decade, the rate of erectile dysfunction in particular has been increasing in military populations [6].

Other recent research has indicated that military personnel report higher rates of sexual dysfunction compared to civilians of similar age $[7,8]$. In addition to being related to mental and physical health problems, sexual dysfunction is associated with significantly lower quality of life and happiness compared to no sexual dysfunction [4].

Many military personnel experience reduced rates of treatment for various other psychological and physical health problems due to stigma and other barriers to care [9]. However, the sensitive nature of sexual functioning problems further complicates treatment seeking. This complexity is further exacerbated by the global lack of graduate-level training related to sexual dysfunction [10]. A lack of provider training in this area leads to limited knowledge and self-efficacy to effectively assess and address sexual functioning problems, which in turn leads to a lack of needed treatment.

There is a clear need for training related to sexual dysfunction among military populations. For established behavioral health providers, it can be difficult to complete additional postgraduate coursework. Thus, convenient and affordable solutions are needed. In an effort to provide preliminary training in this area, the Sex \& the Military: The Other Invisible Wounds of War project created an education toolkit for behavioral health providers who work with military populations and wish to learn about sexual dysfunction. The toolkit consists of three educational video vignettes and an accompanying workbook that provides background information on each scenario, as well as additional resources for working with military populations. The toolkit allows behavioral health providers to complete a self-paced training in this topic area. Additionally, the toolkit can be used in a workshop or classroom settings and the estimated time to complete the toolkit with a class is three hours. While the video vignettes are located online, the toolkit material are available for download on the project webpage at http://cir.usc.edu/research/research-projects/sexual-functioning. This manuscript outlines the learning objectives of the educational toolkit, describes the content and considerations of three case studies presented in the toolkit, and provides suggestions for implementing the toolkit.

\section{Learning objectives}

The Sex \& the Military Toolkit was developed for social workers but is applicable to other behavioral health practitioners, including nurses and psychologists, among others, who plan to work with military populations or individuals experiencing sexual dysfunction. To provide an introductory framework to increase awareness of this critical issue and offer preliminary tools to increase knowledge related to this topic, the toolkit has the following learning objectives:

1. Recognize salient issues related to intimacy and sexual functioning among military personnel, veterans, and their intimate partners.

2. Identify communication strategies for talking with patients about sexual functioning.

3. Recognize intervention strategies for patients with sexual functioning issues and when to refer individuals to other clinical personnel.

4. Recognize the need for policy change in the area of sexual functioning and the military and identify strategies to champion this change.

To achieve these objectives, the toolkit includes an overview of the

Correspondence to: Sherrie Wilcox, University of Southern California, USA, E-mail: slwilcox@usc.edu

Key words: continuing education, military, sexual dysfunction, toolkit, training, veterans

Received: March 27, 2015; Accepted: August 19, 2016; Published: August 24 2016 
research in this area, presents educational video vignettes that describe potential provider-patient interactions, and provides supporting material for background and discussion. Each video vignette was created by a group of researchers, social workers, and psychologists based on clinical experiences and existing data in this area, including evidence-based knowledge. The three vignette cases included in the toolkit are presented along with clinical considerations. Although these confabulated vignettes are specific to the military, knowledge related to dealing with various sexual functioning problems can be translated to civilian populations.

\section{Vignette: Jake}

In military populations, veterans with PTSD have an increased risk of sexual dysfunction. Specifically, approximately $80 \%$ of veterans with PTSD also report sexual dysfunction $[11,12]$. Moreover, military personnel with PTSD are 30 times more likely to report sexual dysfunction compared to those without PTSD [4]. To treat PTSD, service members have the choice of medical or psychosocial interventions or both (e.g., psychotherapy and psychopharmacology) or no treatment. In many cases, the medication used to treat PTSD (e.g., selective serotonin reuptake inhibitors, or SSRIs) presents a high risk of sexual dysfunction, including erectile dysfunction, lower sexual desire, and orgasm-related difficulties $[13,14]$. In fact, the only two medications approved by the U.S. Food and Drug Administration (FDA) for treating PTSD are SSRIs $[15,16]$.

This vignette features Jake, a 22-year-old former Marine who completed two tours of active duty, one in Iraq and one in Afghanistan. He separated from the Marine Corps as a Lance Corporal (i.e., military pay grade: E3) and is an example of a highly trained and effective individual who is now struggling with the casualties of combat. Specifically, Jake has PTSD and his case highlights the impact of a psychopharmacology intervention and subsequent complications following the diagnosis of PTSD. Jake also displays symptoms indicative of psychosocial challenges related to reintegration, or his return to civilian life from the military [17]. Some research has indicated that the majority of adult men with sexual dysfunction have difficulty forming new relationships [18].

In Jake's case, he would like to have a meaningful relationship with a woman, go out on dates, and feel safe. He also desires an intimate and sexual relationship. However, he is experiencing feelings of suspiciousness and anger and has nightmares. As a result, he seeks mental health services, is diagnosed with PTSD, and receives psychotropic medication for his nightmares and PTSD symptoms. He has a prescription for Prazosin (an adrenergic medication) for his nightmares and Zoloft (an SSRI) for his PTSD-related symptoms. It is not uncommon for service members suffering from PTSD to be prescribed more than one medication [19]. Commonly, service members will be prescribed an SSRI, which is effective at improving mood and irritability, but may not be as effective in decreasing nightmares or other sleep disturbances. Moreover, medications that are not FDA approved for a specific disorder may be used off-label nonetheless for additional symptom control. This is illustrated in Jake's case; he is taking both a SSRI as prescribed for PTSD and a second medication for nightmares.

In certain mental health cases, sexual symptoms are simply a result of psychologically challenging situations, such as loss of job, death of loved one, or low self-esteem. In other cases, the sexual symptoms are biological, related to endogenous neurochemical dysregulation. Other mental health cases involve an interplay between environmental or psychological and biochemical dysfunction [20].

\section{Clinical considerations for Jake}

SSRIs frequently are used to treat various mental health disorders. Most have FDA approval to treat depression, anxiety, and posttraumatic stress-related disorders. Jake is prescribed an SSRI for the treatment of PTSD and experiences the sexual side effect of erectile dysfunction, which is a common occurrence in this class of medication. After experiencing erectile dysfunction during sexual activity with his girlfriend, he makes the decision to discontinue his use of the drug. Shortly after discontinuing the medication, he begins reexperiencing his PTSD symptoms and nightmares.

North American culture features a hesitancy to discuss sexual activity and individuals are even less open to discussing sexual functioning. Even in clinical practice, a certain level of discomfort exists between providers and patients regarding discussions of sexual functioning, which creates a barrier for diagnosis and treatment. Treatment adherence and outcomes are diminished when sexual dysfunction related to treatment exists. Patients often stop taking medication after sexual functioning problems persist, which may relieve the sexual dysfunction but often creates a reoccurrence of the primary symptoms being treated $[13,21]$.

\section{Pharmacotherapy}

There are rules and regulations related to psychotropic medication in the military. Service members are monitored regularity with respect to the class of drug and the length of treatment. SSRIs are used frequently and are considered a first-choice class of medication for several mental health-related disorders. Besides reported sexual dysfunction, other issues such as weight gain, drowsiness, and increased suicidal ideation are also problematic.

Service members are very knowledgeable about the fitness requirements and recognize the consequences of being jobless if they fail to meet those requirements. An increase in weight as a result of using SSRIs is not a tolerable side effect and often leads to premature discontinuation of the medication. This is also true when sexual dysfunction occurs. In such cases, it is common for the service member to stop using the medication without the knowledge of the prescriber, or the prescriber may not find out until weeks after the service member stops taking the medication. Other medication-related issues may compromise the efficacy and competence of the service member. For example, symptoms of drowsiness can be incompatible with primary duties related to military service, such as driving or the requirement to carry or operate a weapon. The risk of an increase in suicidal ideation is a major concern, especially in today's military climate of war and combat, which increases the likelihood that a service member has access to weapons.

\section{Sex-specific interventions}

There are several options for treatment of sexual dysfunction resulting from medications (e.g., SSRIs) that can be discussed with the prescribing physician. Dose reduction is an option that may allow the patient to continue using the medication and associated reductions in primary symptoms while potentially improving sexual functioning. If the dose cannot be reduced, there is the option of adding concomitant medications, such as phosphodiesterase-5-inhibitors (e.g., Sildenafil, Vardenafil, and Tadalafil), Viagra, Levitra, or Cialis. These medications have the potential to improve sexual functioning while maintaining the benefits of the medication. Patients can also take a "drug holiday" [22] 
during which the patient consciously decides to not take the prescribed medication on a specific date in anticipation of a sexual experience. Drug holidays often are planned for the weekend or a special event, such as a wedding anniversary.

\section{Concluding remarks for Kake}

A clear and concise informed consent is needed. This consent must address the risk and benefits of treatment and include the option of no treatment. Moreover, the service member must be aware of and be able to articulate his or her understanding of possible increases in suicidal risk, weight gain, and sexual dysfunction. This discussion must also include a plan of action if or when these side effects occur. Psychotropic intervention must also include a start and stop date. In other words, the service member must be aware how long he or she will be taking the medication. An overall review of possible side effects, treatment options, and clinical expectations will likely increase compliance and improve overall treatment outcomes.

\section{Vignette: Grace}

More women have been joining the military and are present in combat zones. As such, women are exposed to the risk of physical and psychological injuries and death. Potentially permanent bodily changes that can result from a combat-related injury can be predictive of poor sexual satisfaction and negative self-esteem [23]. Moreover, the psychological sequelae of a physical injury, including body image dissatisfaction and depression, can interfere with relationships and lead to sexual functioning problems [24]. Injury victims who use mental disengagement as a coping mechanism have reported greater body image dissatisfaction and poor social adjustment [25]. Moreover, whether or not a physical injury exists, female veterans with PTSD or depression have a much higher risk of sexual dysfunction, including reduced desire and difficulty experiencing orgasm, compared to those without PTSD [11,26-32].

This vignette presents a same-sex interracial couple (both age 31) that is dealing with Grace's adjustment to home life after sustaining injuries to her legs from an improvised explosive device (IED) blast. Grace was an Air Force officer serving as a linguist. She was on her way to a village when her translator stepped on an IED, which also injured her. Although Grace did not lose any limbs, she experienced severe burns on her lower body resulting in reduced body image satisfaction, depression, and possibly PTSD, in addition to anorgasmia. She has been with her partner, Claire, for 9 years since meeting in college.

\section{Clinical considerations for Grace}

This vignette highlights issues of body image and the effects of trauma on couples. In the vignette, Claire wants to resume sexual relations as a means of reconnecting emotionally but is unrealistic in her expectations of Grace's adjustment. The trigger for an explosive outburst from Grace comes when Claire has planned a vacation and expects Grace to wear a bikini, seemingly oblivious to Grace's feelings about the scars on her legs. Although Grace has only been home for 3 weeks Claire expects her partner to have adjusted already, stating: "She should be happy she's home." Claire's strategy of using sex to reconnect backfires because Grace is preoccupied and unable to engage in the experience. The film highlights the fact that many factors contribute to a positive sexual experience, including psychosocial and biological.

For her part, Claire has missed Grace and wants to pick up where they left off. She appears to be in total denial that Grace has been traumatized in both body and spirit. Her efforts to connect sexually have a desperate quality to them, reflecting her own losses during Grace's deployment. She may be using Grace (and sex) to avoid dealing with painful feelings of loneliness, emptiness, anger, and fear.

Grace's scars are only the outward manifestation of her internal wounds. Inculcated with the military philosophy of stoicism, Grace had a self-image as a strong, invulnerable woman that has been shattered. Forced to recognize this vulnerability every time she looks at her legs, the shame she experiences results in her desire to withdraw and hide, even from the people who care for her the most. Grace's inability to enjoy her relationship with her partner can be conceptualized from a Cognitive Behavioral perspective. Grace has a negative cognitive set with respect to her body specifically due to IED blast, which created the burns and disfiguration to her legs. As a result, her self-perception and her ability to be fully intimate with her partner are impaired. Grace is pulling away from Claire, both emotionally and physically. Grace is consumed by negative thoughts regarding her self-image which is essentially shutting down her sexual desire and arousal and result in the orgasmic dysfunction implied in the video vignette as well.

The military is characterized above all as a masculine culture. Thus, even though Grace is a woman, she has been steeped in this tradition, which requires her to stoically internalize her emotions. Although this strategy may work during military service, it is counterproductive in relationships, in which communication is critical to emotional connection.

\section{Therapy}

Cognitive restructuring, also known as cognitive reframing can be useful in challenging her negative automatic thoughts. This initial approach may help Grace reframe her perception of her leg injuries (Burns due to an explosion) and allow her to re-engage with Claire. Then Gradual Exposure Therapy can be used to address the traumatic effects of the explosion she experienced while deployed as well as desensitizing her shame related to intimacy with Claire (touching her legs). Regarding the latter, Grace might begin a sexual encounter wearing sweatpants and gradually reduce the amount of clothing she wears to bed.

It might also be helpful for Grace to share her deployment experiences with her partner in joint sessions, to help Claire gain a better understanding of the issues with which Grace is wrestling. Claire would also benefit from some psychoeducation regarding posttrauma adjustment. Her window of 3 weeks is unrealistic, putting unnecessary pressure on Grace. Her expectations have a "get over it" quality, which may exacerbate the shame Grace already feels.

However, Claire's feelings must also be acknowledged. The needs of military partners are often minimized yet their service is just as valuable as the person who is deployed. It would be helpful for Grace to gain a better understanding of Claire's experience as well, perhaps later in therapy when she is more emotionally open to hearing it.

The unique characteristics of this couple should also be addressed. As a same-sex and interracial couple, Grace and Claire may have issues that have not been acknowledged in the past but that may be contributing to underlying tensions. Issues such as internalized homophobia, levels of acceptance and support from their extended family, and political affiliations of other individuals in their community are some of the most salient factors to be considered.

\section{Sex-specific interventions}

Because sex is not the primary issue in this case and seems to be 
putting added pressure on Grace, we would suggest taking a break from sex [33]. Sex might resume gradually at Grace's initiation and pace. Modified sensate-focus techniques or cuddling and other nongenital affectionate gestures are indicated. It is also possible that sex has some deeper meanings now for Grace related to her military experience. For example, was she sexually assaulted? Many of these incidents go unreported or not believed [34]. If they occurred, Grace may have suppressed such incidents to manage her military service. Other possibilities are discussed in the subsequent vignette.

\section{Concluding remarks for Grace}

This video highlights various challenges, including body image concerns, internalizing emotions, and communication challenges, among others. Both partners are experiencing different types of issues. Grace is struggling to deal with her scars and Claire is experiencing challenges reconnecting with her partner. In many cases, partners of service members who have returned from a deployment often experience rejection sensitivity when they don't receive their desired communication from their partner [35]. Overall, the strategies applied to Grace and Claire emphasize individualized therapy, couples therapy, and psychoeducation. These strategies are also applicable to civilian populations.

\section{Vignette: Manny}

Genital injuries are common in the post-9/11 generation of combat veterans, largely due to the increased number of patrols conducted on foot and high exposure to explosive devices [36,37]. An estimated 5\% to $12 \%$ of injuries in the post-9/11 era have involved genital injuries $[38,38]$. These injuries can result in pain during sexual intercourse $[39,40]$ and feelings of emasculation and may lead to difficulties with intimacy [41]. This combination of effects can lead to avoidance of sexual activities with a partner [42] and may lead to other methods of sexual gratification, such as pornography use, which is associated with feelings of betrayal and negative self-esteem in sexual partners $[43,44]$

Manny is a 36-year-old former Army staff sergeant who is married to his high school sweetheart, Angela, age 35. During their 8-year marriage, Manny has been deployed four times. During his last deployment he sustained a urotrauma injury from an IED blast and one of his testicles had to be removed. Urogenital and pelvic pain is associated with sexual dysfunction, including erectile dysfunction and reduced sexual desire $[45,46]$. Although it has been 8 months since his surgery, Manny continues to feel some pain and sensitivity in the affected area, especially during sexual contact. Pain during sex can negatively affect quality of life and emotional well-being and contribute to psychological effects such as anxiety and depression [42].

\section{Clinical considerations}

This vignette highlights the complexity of intimacy when physiological and psychological factors collide and the overlay of military culture influences the outcome. Manny's inability to confide in his wife about his pain during sex results in avoidance of physical intimacy, stirring up her own insecurities related to getting older, causing her to question her own attractiveness. The hypermasculine nature of military culture and Manny's Latino culture are most likely exacerbating his stoicism and reticence to show any weakness to his wife. Instead, he performs sexually to please her and avoid admitting his limitations to both her and himself.

Manny copes by using pornography, which satisfies him sexually but not emotionally. However, the lack of emotionality is also a benefit such that pornography does not place any emotional demands on him. Emotional connection might be difficult for him in this posttrauma era. Additionally, through masturbation he can test his new body, discovering what feels good and what he needs to avoid. Angela, however, feels hurt by Manny's use of pornography, which can lead to feelings of betrayal, negative self-esteem, and sexual dissatisfaction $[43,44]$.

Manny's warrior mentality also contributes to his problems. He expects himself to always be in control and in charge, yet he cannot control his pain. Manny feels as though he is being attacked by his body, but he is unable to fight back and ends up withdrawing from his wife. This feels like surrender, an unacceptable state. His warrior mentality is further challenged by the fact that he lost a testicle, one of his "man parts." Thus, he feels like literally less of a man and, as with all narcissistic injuries, experiences shame.

Sexuality might have additional meanings for Manny. Nudity might trigger intrusive thoughts such as memories of fellow soldiers lying mutilated or dead, with clothes torn off and bleeding. Survivor guilt might also play a role in sexual inhibition. How can he allow himself to enjoy the pleasures of physical intimacy when his best friends are no longer alive? This perfect storm of biological, psychological, social, and cultural factors all contribute to Manny's sexual impairment.

Angela has played the role of the good, supportive military wife. She feels she has "also served" and is angry that Manny hasn't fully returned to her. It could not have been easy to continuously rearrange her life during his comings and goings. Family boundaries needed to contract to accommodate his absence and expand again upon his return. Multiple deployments put a strain on the family system, yet Angela appears to have managed well. She feels entitled to be rewarded by having her husband again.

\section{Therapy}

The key to successful therapy in this case involves communication $[8,47,48]$. In couples therapy, Manny and Angela would share their insecurities and fears with each other. Both worry about not being attractive to the other-Manny because of his deformity and Angela because of aging. As they share their vulnerabilities with each other, they would deepen their relationship. Manny might also benefit from some individual sessions to work through the trauma of the blast, the resulting consequences to his body, and the meaning of the loss of a testicle. His avoidance of intimacy with Angela might also indicate some PTSD symptomatology. This diagnosis should be considered. It is also possible that with time the sensitivity and pain that Manny is currently experiencing may subside. A referral to an occupational therapist might be advisable to speed up this process. He may be attributing an inaccurate meaning to this loss, such as that he is less of a man, an irrational belief that may be contributing to his sexual issues.

\section{Sex-specific interventions}

Communication is also necessary for the couple to develop a new sexual repertoire. Positions and activities they may have enjoyed in the past might not work now with Manny's injuries. He may need a lot of encouragement to give Angela feedback in the moment regarding what feels good to him and what causes discomfort. A therapist might suggest that they see themselves as explorers, going on a sexual expedition to discover new areas of eroticism. This could be done through various nonverbal communication strategies such as sensual massage and individual self-pleasuring [33]. 


\section{Concluding remarks for Manny}

Sexual functioning problems are complex. Manny's case involves various challenges, including physical injury, fear of pain, and lack of control, among others, and frustration was present in both partners. The suggested strategies emphasize communication and exploration. Strategies that can be applied to Manny are also relevant to civilian populations that are experiencing similar challenges.

\section{Conclusions}

Few opportunities exist to provide behavioral health providersin-training and established providers with specialized training in sexual dysfunction, and even fewer resources are available for those who intend to work with military populations. The Sex \& the Military Toolkit provides an introductory overview of the topics of erectile dysfunction, medication-induced sexual dysfunction, anorgasmia, and pornography use, among others. The toolkit provides behavioral health providers with preliminary insight into how a clinical interaction related to sexual functioning may proceed and how the story told at home may differ from what is being described to the provider. Various medical and psychosocial interventions, communication strategies, and policy considerations are also presented.

The toolkit was designed for use in a classroom or workshop, but is also suitable for self-study. We hope that this is the first initiative among many to bring this hidden issue and associated clinical skills and considerations into the provider's repertoire. After all, if a provider is unable to address various topics that may arise in a clinical setting, then those issues may go untreated. To ensure that military and civilian populations have their sexual functioning problems adequately addressed, behavioral health providers need to be prepared to address these topics. However, they do not need to be an expert, and most importantly must know when to refer clients to a specialist. The Sex \& the Military Toolkit is available online at http://cir.usc.edu.

\section{References}

1. U.S Office of the Surgeon General (2001) The surgeon general's call to action to promote sexual health and responsible sexual behavior. [Crossref]

2. World Health Organization (2014) Sexual and reproductive health: Defining sexual health. Wright KM, Britt TW, Moore D (2014) Impediments to mental health treatment as predictors of mental health symptoms following combat. Journal of Traumatic Stress 27: 535-541.

3. Satcher D (2001) The surgeon general's call to action to promote sexual health and responsible sexual behavior. Am J Psychiatry 32: 356-368. [Crossref]

4. Wilcox SL, Redmond S, Hassan AM (2014) Sexual functioning in military personnel: preliminary estimates and predictors. $J$ Sex Med 11: 2537-2545. [Crossref]

5. Mulligan T, Moss CR (1991) Sexuality, aging in male veterans: a cross-sectional study of interest, ability, and activity. Arch Sex Behav 20: 17-25. [Crossref]

6. Armed Forces Health Surveillance Center (2014) Erectile dysfunction among male active component service members US Armed Forces 2004-2013. MSMR 21(9): 13-16. [Crossref]

7. Breyer BN, Cohen BE, Bertenthal D, Rosen RC and Neylan TC, et al., (2014) Sexual dysfunction in male Iraq and Afghanistan war veterans: Association with posttraumatic stress disorder and other combat-related mental health disorders: A population-based cohort study. J Sex Med 11: 75-83. [Crossref]

8. Byers ES, MacNeil S (1997) The relationships between sexual problems, communication, and sexual satisfaction. Canadian Journal of Human Sexuality 6: 277-283.

9. Turchik JA, Bucossi MM, Kimerling R (2014) Perceived barriers to care and gender preferences among veteran women who experienced military sexual trauma: Psychol Serv 2: 180-188. [Crossref]

10. Hough S, Squires LE (2012) Recruitment, retention and professional development of psychologists in America: Potential issue for training and performance. Sexuality and Disability 30: 161-170.

11. Cosgrove DJ, Gordon Z, Bernie JE, Hami S, Montoya D, et al., (2002) Sexual dysfunction in combat veterans with post-traumatic stress disorder. Urology 60: 881884. [Crossref]

12. Letourneau EJ, Schewe PA, Frueh BC (1997) Preliminary evaluation of sexual problems in combat veterans with PTSD. J Trauma Stress 10: 125-132. [Crossref]

13. Bonierbale M, Tignol J (2003) The ELIXIR study: Evaluation of sexual dysfunction in 4557 depressed patients in France. Curr Med Res Opin 19: 114-124. [Crossref]

14. Rosen RC, Lane RM, Menza M (1999) Effects of SSRIs on sexual function: A critical review. J Clin Psychopharmacol 19: 67-85. [Crossref]

15. Brady K, Pearlstein T, Asnis GM, Baker D and Rothbaum B, et al., (2000). Efficacy and safety of sertraline treatment of posttraumatic stress disorder: A randomized controlled trial. JAMA 283: 1837-1844. [Crossref]

16. Marshall RD, Beebe KL, Oldham M, Zaninelli R (2001) Efficacy and safety of paroxetine treatment for chronic PTSD: a fixed-dose placebo-controlled study. Am J Psychiatry 158: 1982-1988. [Crossref]

17. Wilcox SL, Oh H, Redmond SA, Chicas J, Hassan AM, et al., (2015) A scope of the problem: Post-deployment reintegration challenges in a National Guard Unit. Work 50 73-83. [Crossref]

18. Guest JF and Gupta RD (2002) Health-related quality of life in a UK-based population of men with erectile dysfunction. Pharmacoeconomics, 20: 109-117. [Crossref]

19. Davidson J (1992) Drug therapy of post-traumatic stress disorder. Br J Psychiatry 160: 309-314. [Crossref]

20. Preston JD, O’Neal JH, Talaga MC (2013) Handbook of clinical psychopharmacology for therapists (7th ed.) Oakland CA: New Harbinger.

21. Scranton RE, Goldstein I, Stecher VJ (2013) Erectile dysfunction diagnosis and treatment as a means to improve medication adherence and optimize comorbidity management. J Sex Med 10(2): 551-561. [Crossref]

22. Rothschild AJ (1995) Selective serotonin reuptake inhibitor-induced sexua dysfunction: Efficacy of a drug holiday. American Journal of Psychiatry 152: 1514 1516. [Crossref]

23. Tudahl LA, Blades BC, Munster AM (1987) Sexual satisfaction in burn patients. J Burn Care Rehabil 8: 292-293. [Crossref]

24. Fauerbach JA, Heinberg LJ, Lawrence JW, Munster AM, Palombo DA, et al., (2000) Effect of early body image dissatisfaction on subsequent psychological and physical adjustment after disfiguring injury. Psychosom Med, 62: 576-582. [Crossref]

25. Fauerbach JA, Heinberg LJ, Lawrence JW, Bryant AG and Richter L, et al., (2002) Coping with body image changes following a disfiguring burn injury. Health Psychol 21: 115-121. [Crossref]

26. Atlantis E, Sullivan T (2012) Bidirectional association between depression and sexual dysfunction: A systematic review and meta-analysis. J Sex Med 9: 1497-1507. [Crossref]

27. Cohen BE, Maguen S, Bertenthal D, Shi Y, Jacoby V, et al., (2012) Reproductive and other health outcomes in Iraq and Afghanistan women veterans using VA health care: Association with mental health diagnoses. Womens Health Issues 22: e461-e471. [Crossref]

28. De Silva P (2001) Impact of trauma on sexual functioning and sexual relationships. Sexual and Relationship Therapy 16: 269-278.

29. Derogatis LR, Meyer JK and King KM (1981) Psychopathology in individuals with sexual dysfunction. Am J Psychiatry 138: 757-763. [Crossref]

30. Johnson SM, Makinen JA, Millikin JW (2001) Attachment injuries in couple relationships: a new perspective on impasses in couples therapy. J Marital Fam Ther 27: 145-155. [Crossref]

31. Meis LA, Erbes CR, Polusny MA, Compton JS (2010) Intimate relationships among returning soldiers: The mediating and moderating roles of negative emotionality, PTSD symptoms, and alcohol problems. J Trauma Stress 23: 564-572. [Crossref]

32. Williams K, Reynolds MF (2006) Sexual dysfunction in major depression. CNS Spectr 11: 19-23. [Crossref]

33. Maltz W, Christiansen S, Joffee G (1995) Relearning touch: Healing techniques for couples [DVD]. Eugene OR: Intervision Media.

34. Zaleski KL, Katz LS (2014) Alice in wonderland: Exploring the experiences of female 
service members with a pregnancy resulting from rape. Social Work in Mental Health 12: $391-410$.

35. Calhoun PS, Beckham JC, Bosworth HB (2002) Caregiver burden and psychological distress in partners of veterans with chronic posttraumatic stress disorder. J Trauma Stress 15: 205-212.[Crossref]

36. Sharma DM, Webster CE, Kirkman-Brown J, Mossadegh S, Whitbread T, et al., (2013) Blast injury to the perineum. $J$ R Army Med Corps 159: i1-i3. [Crossref]

37. Waxman S, Beekley A, Morey A, Soderdahl D (2009) Penetrating trauma to the external genitalia in Operation Iraqi Freedom. Int J Impot Res 21: 145-148. [Crossref]

38. Woodward C, Eggertson L (2010) Homemade bombs and heavy urogenital injuries create new medical challenges. Canadian Medical Association Journal 182: 11591160.

39. Morey AF, Metro MJ, Carney KJ, Miller KS, McAninch JW, et al., (2004) Consensus on genitourinary trauma: External genitalia. BJU Int, 94, 507-515. [Crossref]

40. Wesselmann U, Burnett AL, Heinberg LJ (1997) The urogenital and rectal pain syndromes. Pain 73: 269-294. [Crossref]

41. Frappell-Cooke W, Wink P, Wood A (2013) The psychological challenge of genital injury. J R Army Med Corps 159 Suppl 1: i52-56. [Crossref]
42. Corden C (2013) Causes and management of dyspareunia. InnovAiT 6: 66-75.

43. Stewart DN, Szymanski DM (2012) Young adult women's reports of their male romantic partner's pornography use as a correlate of their self-esteem, relationship quality, and sexual satisfaction. Sex Roles 67: 257-271.

44. Zitzman ST (2007) Pornography viewing as attachment trauma in pair-bond relationships: A theoretical model of mechanisms (Doctoral dissertation). Brigham Young University Provo UT.

45. Lutz MC, Roberts RO, Jacobson DJ, McGree ME, Lieber MM, et al., (2005) Crosssectional associations of urogenital pain and sexual function in a community based cohort of older men: Olmsted County, Minnesota. J Urol 174: 624-628. [Crossref]

46. Mehik A, Hellström P, Lukkarinen O, Sarpola A, Järvelin M et al., (2000) Epidemiology of prostatitis in Finnish men: a population-based cross-sectional study. BJU Int 86: 443-448. [Crossref]

47. Markman HJ, Renick MJ, Floyd FJ, Stanley SM, Clements M et al., (1993) Preventing marital distress through communication and conflict management training: A 4- and 5-year follow-up J Consult Clin Psychol 61: 70-77. [Crossref]

48. Sherman MD, Zanotti DK, Jones DE (2005) Key elements in couples therapy with veterans with combat-related posttraumatic stress disorder. Professional Psychology: Research and Practice 36: 626-633.

Copyright: $\odot 2016$ Wilcox SL. This is an open-access article distributed under the terms of the Creative Commons Attribution License, which permits unrestricted use, distribution, and reproduction in any medium, provided the original author and source are credited. 